\title{
Intoxicação experimental por Trema micrantha (Ulmaceae) em bovinos ${ }^{1}$
}

\author{
Sandra Davi Traverso ${ }^{2}$, André Mendes Ribeiro Corrêa ${ }^{2}$, Milene Schmitz ${ }^{2}$, Edson \\ Moleta Colodel ${ }^{2}$ e David Driemeier ${ }^{2}$
}

\begin{abstract}
Traverso S.D., Correa A.M.R., Schmitz M., Colodel E.M. \& Driemeier D. 2004 [Experimental poisoning by Trema micrantha (Ulmaceae) in cattle.] Intoxicação experimental por Trema micrantha (Ulmaceae) em bovinos. Pesquisa Veterinária Brasileira 24(4):211-216. Setor de Patologia Veterinária, Depto Patologia Clínica Veterinária, Faculdade de Veterinária, UFRGS, Av. Bento Gonçalves 9090, Porto Alegre, RS 91540-000, Brazil. E-mail: davetpat@vortex.ufrgs.br

Leaves of Trema micrantha were orally given to 13 cattle. Ten animals received green leaves in a single dose, two animals received green leaves in fractionated doses, and one received the dried leaves in a single dose. Eight animals showed clinical signs and six of them died. Clinical signs were observed 16 hours after administration and included apathy, anorexia, drooling, progressive weakness, coma and death. Neurological signs as pressing the head against obstacles and head shaking were observed in four animals . Death occurred between 67 and 153 hours after the end of plant ingestion. The main gross lesions were observed in the liver, and included friable consistency, pronounced lobular pattern and areas of haemorrhages. The liver of one bovine was homogeneously dark reddened. Petechial hemorrhages in serosal membranes and edema in the gall bladder were frequently seen. Pale kidneys with red spots in the cortex were observed in one animal. Microscopically, the most striking lesion in the liver was massive coagulative necrosis, associated with centrolobular haemorrhages, observed in four animals. In the liver of one bovine centrolobular necrosis was observed . Tubular renal necrosis was noted in two animals. Additional microscopic lesions were found in the central nervous system of five bovines, especially in the frontal cortex, and included perineuronal and perivascular edema with basophilia and retraction of the neurons. T T. micrantha caused clinical signs with $50 \mathrm{~g} / \mathrm{kg}$ and death with doses of $54 \mathrm{~g} / \mathrm{kg}$ or higher. The fractionated administration of the green leaves as well as the dried leaves did not cause poisoning.
\end{abstract}

INDEX TERMS: Poisonous plants, plant poisoning, Trema micrantha, Ulmaceae, cattle, liver necrosis.

RESUMO.- Folhas de Trema micrantha foram fornecidas a 13 bovinos. Dez deles receberam folhas verdes em dose única, dois receberam folhas verdes em doses fracionadas e um recebeu dose única de planta dessecada. Oito dos bovinos apresentaram sinais clínicos e seis morreram. Os sinais clínicos iniciaram a partir de 16 horas após a ingestão da planta. Os animais apresentavam apatia, anorexia, sialorréia, fraqueza progressiva, coma

\footnotetext{
${ }^{1}$ Recebido em 2 de julho de 2004.

Aceito para publicação em 20 de julho de 2004.

2 Setor de Patologia Veterinária, Depto Patologia Clínica Veterinária, Faculdade de Veterinária, Universidade Federal do Rio Grande do Sul (UFRGS), Av. Bento Gonçalves 9090, Porto Alegre, RS 91540-000. *Autor para correspondência. E-mail: davetpat@vortex.ufrgs.br
}

e morte. Sinais neurológicos, tais como pressionar a cabeça contra obstáculos e movimentos laterais da cabeça, foram observados em quatro animais. As mortes ocorreram entre 67 e 153 horas após o término da ingestão da planta. $\mathrm{O}$ achado de necropsia mais significativo foi observado no fígado que era friável com padrão lobular acentuado e com áreas de hemorragia, exceto um bovino, que apresentou o fígado difusamente vermelho escuro. Hemorragias petequiais nas serosas e edema da vesícula biliar foram alterações adicionais observadas com freqüência. Rins pálidos com pontos avermelhados na cortical foram observados em um bovino. $O$ exame histológico revelou, em 5 animais, necrose de coagulação massiva associada à hemorragia centrolobular acentuada. Um animal apresentou necrose centrolobular. Dois animais apresentaram alterações nos 
rins, caracterizadas por necrose tubular renal. Altrações histológicas no sistema nervoso central, que consistiam de edema perineuronal e perivascular com basofilia e retração de neurônios, principalmente no córtex frontal, foram observadas em cinco bovinos. T. micrantha causou sinais clínicos com $50 \mathrm{~g} /$ $\mathrm{kg}$ e morte a partir de $54 \mathrm{~g} / \mathrm{kg}$ de peso. As doses fracionadas e a planta dessecada não causaram sinais de intoxicação.

TERMOS DE INDEXAÇÃO: Plantas tóxicas, intoxicação por planta, Trema micrantha, Ulmaceae, bovinos, necrose hepática.

\section{INTRODUÇ̃̃O}

O gênero Trema inclui espécies de árvores de crescimento rápido que são utilizadas como pioneiras em sistemas de reflorestamento depois de queimadas e na recuperação ambiental de áreas degradadas (Nowotny \& Nowotny 1993, Castellani \& Aguiar 1998).

A planta é palatável e intoxicação natural por Trema micrantha foi observada em caprinos após o fornecimento da planta na alimentação ( Gava, comunicação pessoal 2000, Traverso et al. 2003). Recentemente, após o corte de uma árvore numa pastagem com cabras, 7 animais que estavam no piquete morreram com necrose hepática após a ingestão de folhas da planta (Traverso \& Driemeier, dados não publicados 2004). A intoxicação foi reproduzida em caprinos e coelhos (Traverso \& Driemeier 2000, Traverso et al. 2002). O quadro clínico observado é de insuficiência hepática por necrose hepatocelular (Traverso et al. 2002, 2003). Outras alterações histológicas relatadas em caprinos e coelhos foram edema perineuronal e perivascular, tumefação e necrose de neurônios, no córtex cerebral e hipocampo (Traverso \& Driemeier 2000, Traverso et al. 2002) e degeneração hidrópica do epitélio tubular renal (Traverso et al. 2002).

No Brasil, quadros de insuficiência hepática aguda foram relatados nas intoxicações naturais por Cestrum laevigatum (Döbereiner et al. 1969), Cestrum parqui (Riet-Correa et al. 1986), C. corymbosum var. hirsutum (Gava et al. 1991), C. intermedium (Gava et al. 1996), Sessea brasiliensis (Canella et al. 1968), Vernonia mollissima

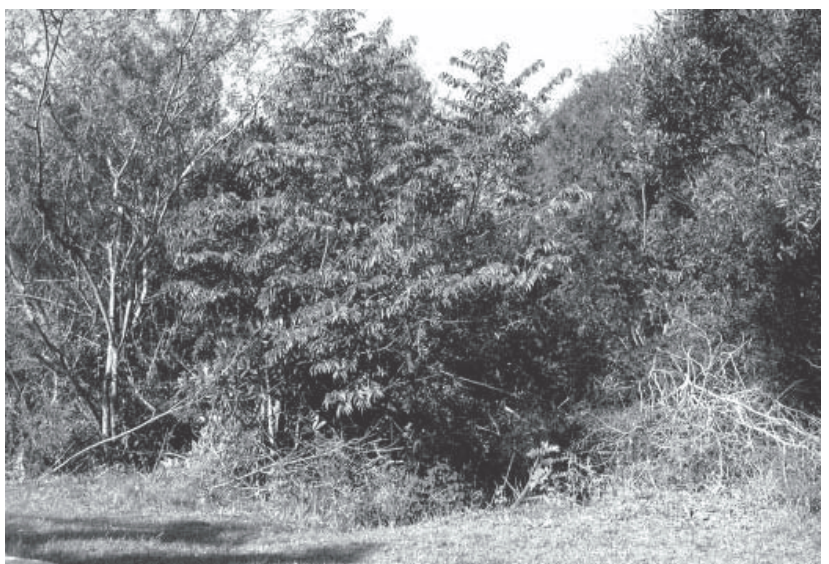

Fig. 1. Trema micrantha de porte médio em crescimento, no mês de junho.
(Döbereiner et al. 1976), Vernonia rubricaulis (Tokarnia \& Döbereiner 1982, Brum et al. 2002), Xanthium cavanillesii (Driemeier et al. 1999), Myoporum laetum (Bonel-Raposo et al. 2003) e Dodonea viscosa (Colodel et al. 2003). Como ocorrem quadros clínicos de necrose hepatocelular sem etiologia estabelecida e T. micrantha ocorre em todo território brasileiro, em áreas comuns a essas plantas, há necessidade de diferenciar os quadros clínico e patológico observados na intoxicação por T. micrantha com os de outras plantas hepatotóxicas para bovinos.

\section{MATERIAL E MÉTODOS}

Classificação botânica e coleta. Amostras de Trema micrantha (L.) Blume (Fig. 1) utilizadas no experimento foram encaminhadas à Fundação Zoobotânica do Rio Grande do Sul para classificação morfológica. Folhas verdes de T. micrantha foram coletadas nos municípios de Porto Alegre e Viamão, RS, e conservadas em refrigerador.

Animais experimentais e exames clínicos. Foram utilizados 13 bovinos, com idade de 8 a 36 meses, que receberam folhas de T. micrantha (Quadro 1). Ao Bovino 1 foram administradas as folhas verdes frescas por via oral em dose única, aos demais a planta era pesada e colocada no cocho para a ingestão voluntária. Bovino 9 recebeu folhas dessecadas em temperatura ambiente à sombra por 15 dias; os outros receberam as folhas verdes frescas. A dose foi fracionada para os Bovinos 7 e 8 e foi única para os demais. Os animais foram estabulados em baias de alvenaria, e antes do experimento foram mantidos em jejum prévio de 24 horas. Após a administração da planta recebiam feno de alfafa, ração comercial para bovinos e água a vontade, e eram acompanhados com exames clínicos periódicos que consistiam de avaliação do comportamento clínico, da temperatura corporal e de auscultações cardíaca, respiratória e digestiva.

Coletas de sangue, uma prévia ao experimento (48 horas antes do jejum) e outras posteriormente à administração da planta em intervalos de 24 horas, foram realizadas nos Bovinos 10 e 11, para dosagem sérica de gama-glutamil transferase (GGT), bilirrubina ${ }^{3}$ (direta e indireta), glicose, uréia e amônia ${ }^{4}$. GGT foi determinada pelo teste cinético Szasz modificado (Szasz 1969); as bilirrubinas foram determinadas pelo método Sims-Horn (Sims \& Horn 1958), a

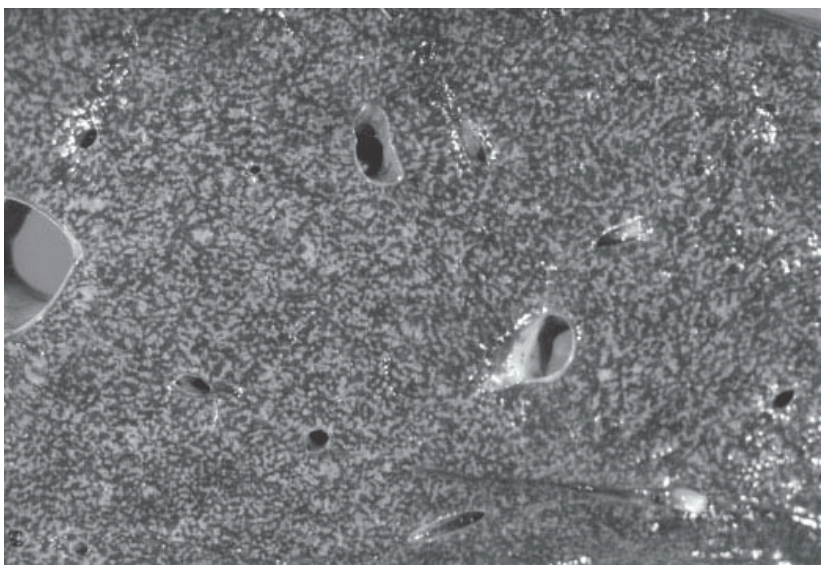

Fig. 2. Superfície de corte de fígado apresenta acentuação do padrão lobular (aspecto "noz moscada") na intoxicação experimental por Trema micrantha (Bovino 1). 
glicose pelo método glicose oxidase (Bergmever 1986) e uréia e amônia foram dosadas pelo método ultravioleta (Bergmever 1985).

Estudo patológico. Os bovinos que morreram, foram imediatamente necropsiados e fragmentos de todos os órgãos, inclusive encéfalo e medula espinhal, foram coletados e fixados em formalina tamponada a 10\% (Prophet et al. 1992). As amostras foram processadas rotineiramente para estudo histopatológico e corados pela técnica de hematoxilina e eosina (HE) (Allen 1992). Nos cortes histológicos dos fígados foi empregada adicionalmente a imunoistoquímica (Mills 1992) com anticorpo anti-citoqueratina ${ }^{5}$ pela técnica de streptavidina biotina na titulação de 1:100, com DAB como cromógeno. Um encéfalo controle, obtido em abatedouro, foi processado simultaneamente com os encéfalos dos casos experimentais. Nos bovinos que não morreram foram realizadas biopsias hepáticas 15 e 30 dias após a administração da planta.

\section{RESULTADOS}

O delineamento, a evolução clínica e o desfecho da intoxicação experimental com folhas de Trema micrantha em bovinos estão apresentadas no Quadro 1. Dos treze bovinos que receberam folhas da planta, oito apresentaram sinais clínicos (Bov. 1, 2, 3, 4 , $5,6,10$ e 11) desses, seis morreram (Bov. 1, 3, 4, 5, 10 e 11) e dois (Bov. 2 e 6) se recuperaram. A quantidade de $50 \mathrm{~g} / \mathrm{kg}$ de peso foi capaz de provocar sinais clínicos e a morte foi observada a partir de $54 \mathrm{~g} / \mathrm{kg}$. Bovino 6 , que recebeu $80 \mathrm{~g} / \mathrm{kg}$, apresentou sinais clínicos e se recuperou. As mortes ocorreram entre 67 e 153 horas após o término da ingestão da planta. Os Bovinos 7 e 8 que receberam doses fracionadas, Bovino 9 que recebeu planta dessecada e Bovinos 12 e 13 que receberam 35 e 40g/ $/ \mathrm{gg}$, não mostraram sinais clínicos de intoxicação.

Sinais clínicos. Os primeiros sinais clínicos nos bovinos que morreram surgiram entre 16 e 67 horas após a ingestão da

\section{Quadro 1. Delineamento, evolução do quadro clínico desfecho da intoxicação experimental com folhas de Trema micrantha (Ulmaceae) em bovinos}

\begin{tabular}{|c|c|c|c|c|c|c|}
\hline $\begin{array}{c}\text { Bovino } \\
\text { no. }\end{array}$ & $\begin{array}{r}\text { Peso } \\
(\mathrm{kg})\end{array}$ & $\begin{array}{c}\text { Dose } \\
(\mathrm{g} / \mathrm{kg})\end{array}$ & $\begin{array}{l}\text { Número } \\
\text { de doses } \\
\text { diárias }\end{array}$ & $\begin{array}{l}\text { Início dos } \\
\text { sinais clíni- } \\
\text { cos (horas) }\end{array}$ & $\begin{array}{c}\text { Evolução } \\
\text { clínica } \\
\text { (horas) }\end{array}$ & Desfecho \\
\hline 1 & 517 & 54 & única & 16 & 137 & Morreu \\
\hline 2 & 205 & 80 & única & 48 & 72 & Recuperou \\
\hline 3 & 195 & 79 & única & 24 & 99 & Morreu \\
\hline 4 & 216 & 106 & única & 38 & 100 & Morreu \\
\hline 5 & 191 & 57 & única & 20 & 47 & Morreu \\
\hline 6 & 197 & 50 & única & 26 & 48 & Recuperou \\
\hline 7 & 215 & 60 & $1,3 \mathrm{~kg} / \mathrm{d} / 10 \mathrm{dias}$ & is N.A. ${ }^{b}$ & - & NA \\
\hline 8 & 220 & 57 & $500 \mathrm{~g} / \mathrm{d} / 25 \mathrm{dias}$ & N.A & - & NA \\
\hline 9 & 190 & $60^{\mathrm{a}}$ & única & N.A. & - & NA \\
\hline 10 & 86 & 60 & única & 41 & 106 & Morreu \\
\hline 11 & 217 & 60 & única & 67 & 37 & Morreu \\
\hline 12 & 190 & 40 & única & N.A. & - & N.A. \\
\hline 13 & 214 & 45 & única & N.A. & - & N.A. \\
\hline
\end{tabular}

${ }^{3}$ Laboratório de Bioquímica da Faculdade de Veterinária. Av Bento Gonçalves 9090, Porto Alegre, RS.

${ }^{4}$ Instituto H Pardini, Rua Aimorés 33, Belo Horizonte, MG 30140-070.

${ }^{5}$ DAKO Carpinteria, Califórnia. planta. Foram observadas alterações na consistência das fezes que variou de pastosa a líquida, anorexia, apatia e fraqueza progressiva, sialorréia, movimentos mastigatórios vazios e tremores musculares leves, principalmente nos membros anteriores. $\mathrm{O}$ quadro evoluía para relutância ao movimento, decúbito esternal com a cabeça voltada para o flanco, decúbito lateral, movimentos de pedalagem, coma e morte. Notou-se icterícia nos Bovinos 1 , 3, 4 e 5. Os Bovinos 3, 4, 5 e 11 apresentaram também sinais clínicos neurológicos, com movimentos contínuos de estender e retrair o pescoço (Bov. 3 e 4), andar em círculo e bater a cabeça contra a parede (Bov. 4 e 11), hipermetria e posições anômalas de postura (Bov. 4). O Bovino 5 apresentou agressividade e permanecia por longos períodos com alimento na boca sem mastigar, quando movimentado caia várias vezes, permanecendo até 10 minutos em posição de desconforto até retornar a posição de estação. O Bovino 11 tinha dificuldade para beber água, permanecia em decúbito abdominal com a cabeça apoiada no solo ou batendo, por vezes a cabeça no flanco, e tornava a colocá-la no solo fazendo isso repetidas vezes.

Achados laboratoriais. O Bovino 10 apresentou elevação da bilirrubina livre a partir de 24 horas após a ingestão da planta e aumento de GGT e bilirrubina conjugada a partir de 72 horas; não houve alterações significativas no nível sérico de glicose. No Bovino 11, 48 horas após a administração da planta, ocorreu queda nos níveis de glicose e uréia, e aumento progressivo nos níveis de bilirrubina livre e conjugada, da GGT e da amônia. Os níveis de uréia voltaram ao normal no dia da morte do animal. As dosagens séricas realizadas nos Bovinos 10 e 11 estão sumarizadas no Quadro 2.

Achados macroscópicos. Os achados de necropsia mais significativos foram observados no fígado e consistiam de acentuação do padrão lobular nas superfícies capsular e de corte (Fig. 2) caracterizadas por áreas vermelhas escuras, deprimidas, delimitadas por áreas claras e hemorragias aleatórias, exceto o Bovino 11 que apresentava o fígado difusamente vermelho-escuro. Áreas amareladas, circulares, não maiores que $2 \mathrm{~cm}$ eram

Quadro 2. Dosagens séricas da intoxicação por Trema micrantha em bovinos

\begin{tabular}{|c|c|c|c|c|c|c|c|c|}
\hline \multirow{2}{*}{$\begin{array}{c}\text { Animal } \\
\text { no. }\end{array}$} & \multirow{2}{*}{$\begin{array}{l}\text { Hora da } \\
\text { coleta }^{a}\end{array}$} & \multirow{2}{*}{$\begin{array}{c}\text { Glicose } \\
\mathrm{mg} / \mathrm{dl}\end{array}$} & \multicolumn{3}{|c|}{ Bilirrubina } & \multirow{2}{*}{$\begin{array}{c}\text { GGT } \\
\mathrm{u} / \mathrm{i}\end{array}$} & \multirow{2}{*}{$\begin{array}{l}\text { Uréia } \\
\mathrm{mg} / \mathrm{dl}\end{array}$} & \multirow{2}{*}{$\begin{array}{c}\text { Amônia } \\
\mu \mathrm{mol} / \mathrm{l}\end{array}$} \\
\hline & & & $\begin{array}{l}\text { Total } \\
\mathrm{mg} / \mathrm{dl}\end{array}$ & njug & $\begin{array}{l}\mathrm{L} \text { Livre } \\
\mathrm{mg} / \mathrm{dl}\end{array}$ & & & \\
\hline \multirow[t]{7}{*}{ Bovino 10} & 0 & 80 & 1,12 & م & 3 & & & \\
\hline & 24 & 97,6 & 1,6 & 0 , & 5 & 15,6 & 16,6 & 9,7 \\
\hline & 48 & 70,8 & 1,7 & 0,3 & & 17,9 & 13,6 & 3,2 \\
\hline & 7 & 83,7 & 2,6 & 0 , & & 39,2 & 18,6 & 2,1 \\
\hline & 9 & 77,1 & 2,3 & & & 61,9 & 12,2 & 249,8 \\
\hline & 12 & 82,2 & 1,7 & 0 & 2 & 68,9 & 23,5 & 9,2 \\
\hline & 144 & 93,2 & 2,3 & & & 71,1 & 25,8 & 197,3 \\
\hline \multirow[t]{5}{*}{ Bovino 11} & 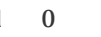 & & & & & 13,3 & 26,6 &, 5 \\
\hline & 24 & 75,2 & 1,61 & 0,23 & 1,38 & 14,1 & 27,4 & 203,9 \\
\hline & 36 & 66,7 & 1,97 & 0 & 1,61 & 23,8 & 22,3 & 225,6 \\
\hline & 48 & 44,6 & 8,49 & 1,23 & 7,26 & 100,7 & 15,1 & 498,7 \\
\hline & 72 & 42,5 & 11,18 & 2,56 & 8,62 & 117,1 & 24,6 & 1250,7 \\
\hline eferênciab & b $45-75$ & $0,01-1,2$ & $<0,4$ & $0,1-1,0$ & $<39$ & $24-42$ & 85,2 & 100,4 \\
\hline
\end{tabular}

a Após a administração da planta.

b Radostits et al. 2000 . 


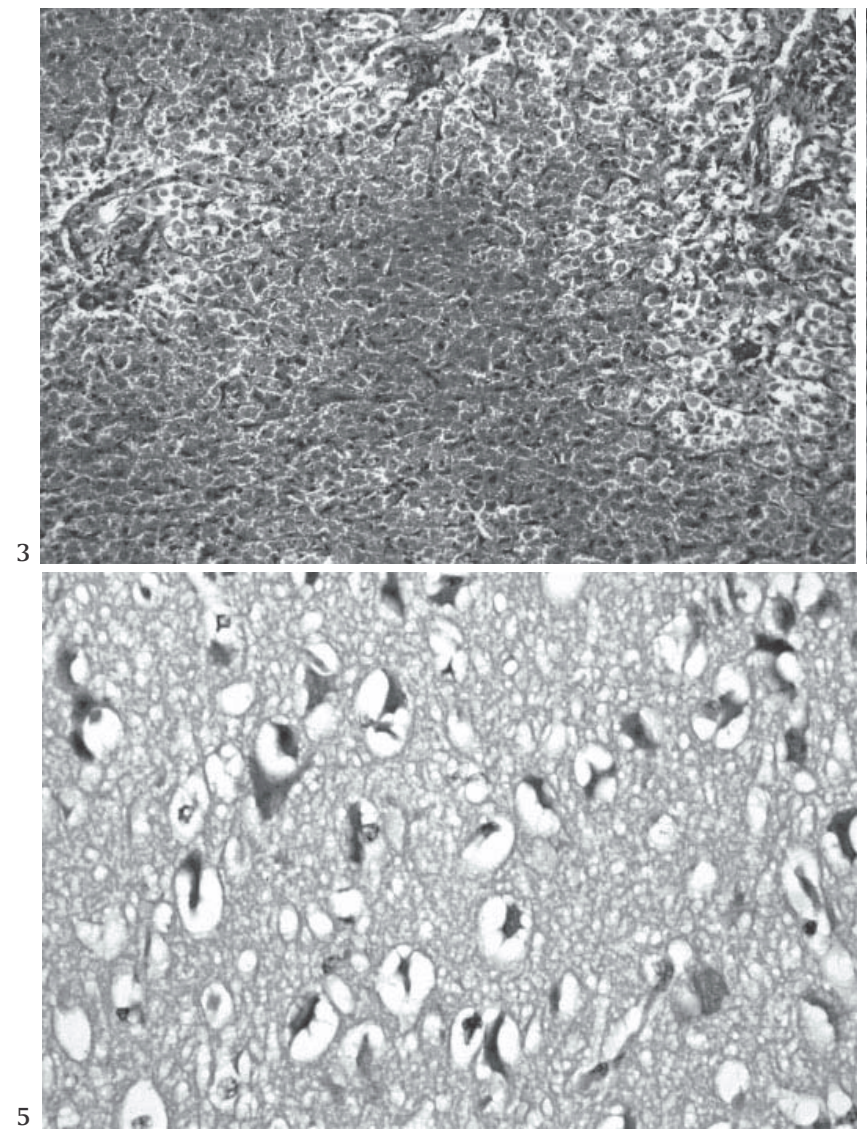

Fig. 3. Necrose hepatocelular maciça e hemorragia centrolobular acentuada na intoxicação experimental por Trema micrantha (Bovino 3). HE, obj. 20.

Fig. 5. Edema perineuronal, basofilia e retração dos neurônios no Córtex frontal, na intoxicação experimental por Trema micrantha (Bovino 3). HE, obj. 40.

observadas na superfície de corte do fígado de todos animais, que apresentaram também petéquias no epicárdio e sufusões no endocárdio, petéquias e sufusões em todas as serosas e todos os órgãos da cavidade abdominal, edema de vesícula biliar e intestino delgado, e reto com fezes ressequidas envoltas por muco e/ou sangue. Ascite foi observada nos Bovinos 5 e 11 . Os Bovinos 1, 5, 10 e 11 apresentavam sangue livre no lúmen intestinal. No Bovino 1 foram observados coágulos livres na cavidade abdominal e na musculatura dorsal aos rins. $\mathrm{O}$ Bovino 3 apresentava no encéfalo um hematoma no lobo parietal esquerdo. Petéquias múltiplas no cerebelo foram observadas no Bovino 5. O Bovino 10 apresentava edema e hemorragia perirrenal, os rins eram pálidos com múltiplos pontos avermelhados na cortical, ao corte havia edema e hemorragia na pelve; edema no teto da cavidade abdominal e em toda cavidade pélvica.

Achados histológicos. Foram observadas lesões hepáticas, nervosas e renais em diferentes intensidades. No figado as necroses observadas caracterizavam-se por desorganização dos cordões de hepatócitos com eosinofilia intensa de citoplasma, e perda de bordos citoplasmáticos, com núcleos em picnose, cariolise e

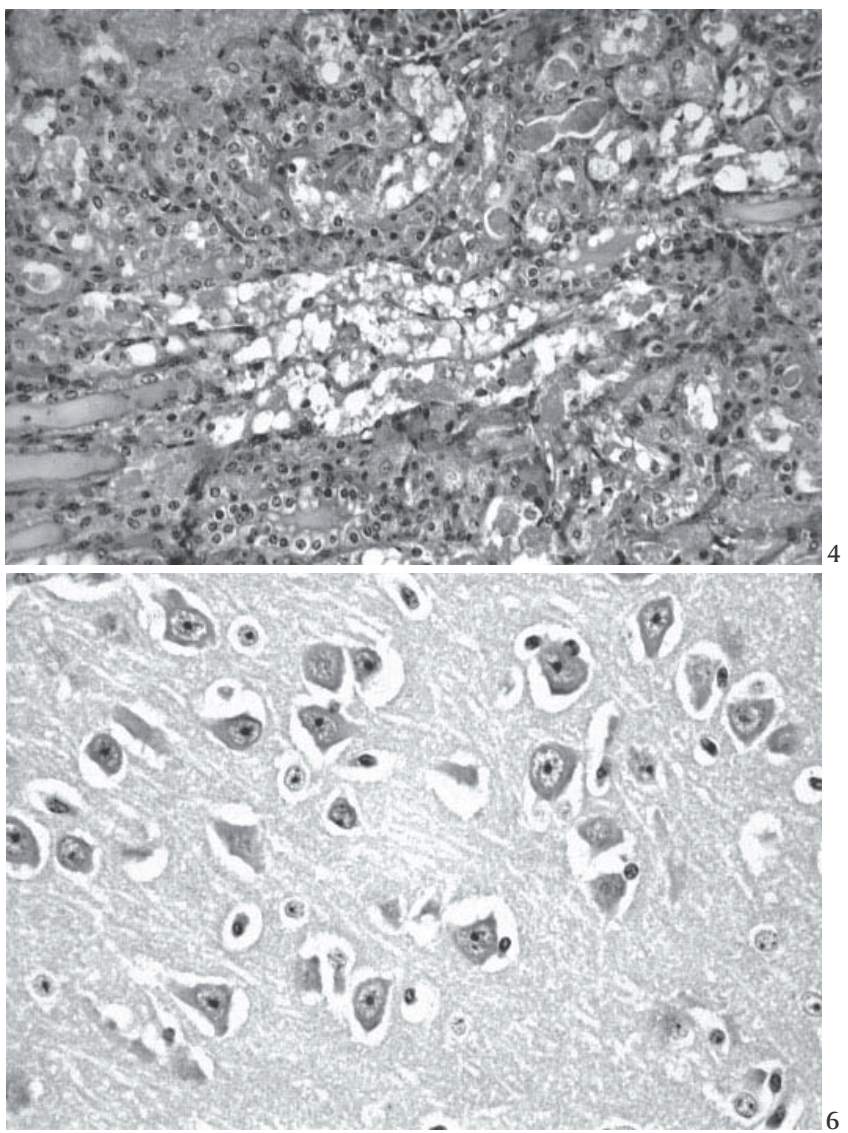

Fig. 4. Necrose do epitélio tubular renal e presença de cilindros hialinos na luz dos túbulos, na intoxicação experimental por Trema micrantha (Bovino 10). HE, obj. 20.

Fig. 6. Córtex frontal normal de encéfalo de bovino controle. HE, obj. 40.

cariorrexia. No fígado dos Bovinos 3, 4, 5 e 11 foi observado necrose hepática maciça, associada a hemorragia centrolobular acentuada (Fig. 3). O Bovino 1 teve necrose centrolobular acentuada e tumefação de hepatócitos portais. Proliferação moderada de ductos biliares foi observada em todos os bovinos, as células proliferadas apresentaram moderada marcação por citoqueratina. Áreas focalmente extensas de hepatócitos tumefeitos e citoplasma espumoso foram observados em todos os animais correspondendo àquelas áreas amareladas, observadas na superfície de corte. Os dois bovinos que adoeceram e se recuperaram (Bov. 2 e 6) e os que não adoeceram (Bov. 7, 8, 9, 12 e 13) não apresentaram alterações histológicas nas biópsias hepáticas.

Lesões renais foram observadas nos Bovinos 10 e 11; no Bovino 10 eram caracterizadas por necrose tubular renal acentuada (Fig. 4), com perda de estrutura tubular, restando grânulos eosinofílicos e debris celulares delimitados pela membrana basal, principalmente nos túbulos do córtex, tumefação de túbulos uriníferos e presença de cilindros hialinos. No Bovino 11 havia degeneração tubular renal caracterizada por tumefação das células tubulares. 
Os Bovinos 1, 3, 4, 10 e 11, que morreram entre 5 e 7 dias após a administração da planta, apresentaram lesões no sistema nervoso central, caracterizadas por edema perineuronal e perivascular (Fig. 5) na substância cinzenta, mais evidente no córtex frontal, as quais não foram observadas no bovinos de controle (Fig. 6). Os Bovinos 3 e 11, apresentaram também neurônios retraídos e basofílicos na região do córtex e no hipocampo, além de basofilia quando comparadas com o controle. O Bovino 5, que morreu em 2 dias e 19 horas após a administração da planta, não apresentou alteração histológica no encéfalo.

\section{DISCUSSÃO E CONCLUSÃO}

Este experimento caracterizou as folhas de Trema micrantha como tóxicas para bovinos, causando morte em doses a partir de $54 \mathrm{~g} /$ $\mathrm{kg}$. Doses iguais ou superiores, quando foram fracionadas ou quando a planta foi dessecada, não demonstraram toxicidade para bovinos. A dose tóxica foi superior àquela observada na intoxicação da mesma planta para caprinos, que era de $30 \mathrm{~g} / \mathrm{kg}$ (Traverso et al. 2002), e de coelhos, que foi de $35 \mathrm{~g} / \mathrm{kg}$ (Traverso \& Driemeier 2000). Não foi observada correlação entre a evolução do quadro clínico e a dose ingerida. A evolução clínica nos bovinos que morreram foi de 37 a 137 horas e nos que se recuperaram foi de 48 a 72 horas. Em caprinos que morreram a evolução clínica não foi superior a 34 horas (Traverso et al. 2002) e em coelhos intoxicados por T. micrantha a evolução clínica variou entre 48 e 120 horas (Traverso \& Driemeier 2000).

As folhas de T. micrantha demonstraram ser hepatotóxicas causando sinais clínicos associados à insuficiência hepática, que foram similares aos descritos para caprinos experimentalmente intoxicados por T. micrantha (Traverso et al. 2002). Nesse trabalho constataram-se alterações bioquímicas pela insuficiência hepática, com elevações da GGT e da bilirrubina, hiperamonemia e hipoglicemia. Possivelmente os sinais clínicos neurológicos observados nos bovinos desse experimento estão relacionados com a encefalopatia hepática (Tennant 1997, Radostits et al. 2000).

Os achados de necropsia, descritos no fígado de bovinos intoxicados por T. micrantha são semelhantes às que ocorrem pelo consumo desta planta em caprinos (Traverso et al. 2002, 2003) e coelhos (Traverso \& Driemeier 2000), bem como nas demais plantas de ação hepatóxica aguda que ocorrem no Brasil (Tokarnia et al. 2000). O edema perirenal observado é semelhante àqueles encontrados nas plantas essencialmente nefrotóxicas, Thiloa glaucocarpa e Amaranthus spp (Tokarnia et al. 2000), e se deve aparentemente ao refluxo tubular, com conseqüente drenagem linfática e saída do líquido até o tecido intersticial perirrenal (Maxie 1993). Não foi possível correlacionar as alterações renais, descritas neste estudo, com a dose ingerida, a evolução clínica ou com o padrão de lesão hepática.

A necrose hepatocelular observada na intoxicação por $T$. micrantha em bovinos é semelhante àquelas descritas para a intoxicação natural e experimental por T. micrantha em caprinos (Traverso et al.2002, 2003) e em bovinos intoxicados por Vernonia spp (Tokarnia et al. 2000),,Dodonea viscosa (Colodel et al. 2003, Cattani et al. 2004), Cestrum corymbosum var. hirsutum (Gava et al. 1991) e Xanthium cavanillesii (Colodel 2000). Notou-se correla- ção entre a intensidade da necrose hepatocelular e a evolução clínica dos bovinos intoxicados por T. micrantha.

As lesões neuronais descritas neste trabalho, caracterizadas por cromatólise neuronal com perdas dos bordos citoplasmáticos e picnose nuclear, relatadas na intoxicação por T. micrantha em coelhos (Traverso \& Driemeier 2000) e caprinos (Traverso et al. 2002, 2003), são similares às descritas em casos de hipoglicemia (Jubb \& Huxtable 1993). Dosagens séricas de glicose, inferiores aos valores por nós observados, foram relatadas nos casos de intoxicação em bovinos por Xanthium cavanillesii (Colodel et al. 2000) e Dodonea viscosa (Colodel et al. 2003); em ambos casos não foram relatadas alterações histológicas no sistema nervoso. Talvez a presença de lesões neuronais nos casos de intoxicação por $T$. micrantha esteja relacionada ao prolongado quadro clínico observado na intoxicação por essa planta. A ausência de lesões neurológicas no Bovino 5, que morreu em 47 horas após o início dos sinais clínicos, corrobora para esta suspeita. A presença de lesões histológicas no sistema nervoso do Bovino 11 , que teve evolução clínica de 37 horas, mas morreu 104 horas após a ingestão da planta, é um indício que o prolongado tempo entre a ingestão da planta até a morte do animal também influencia no desenvolvimento das lesões no sistema nervoso. Em nossas observações e pelos dados obtidos neste trabalho, constata-se que, mesmo com a dose tóxica relativamente alta, podem ocorrer intoxicações naturais em bovinos pelo consumo de T. micrantha após queda da árvore ou quando as folhas são fornecidas como forragem em períodos de estiagem; pois a planta aparenta boa palatabilidade.

\section{REFERÊNCIAS}

Allen T.C. 1992. Hematoxylin and Eosin, p.53-58. In: Prophet E.B., Mills B., Arrington J.B. \& Sobin L.H. (ed.) Laboratory Methods in Histotechnology. Armed Forces Institute of Pathology, Washington, DC. 279p.

Bergmever H.V. 1985. Methods of Enzymatic Analysis. Vol.8. 3rd ed.. Verlag Chemie, Deerfield Beach, p.444-449.

Bergmever H.V. 1986. Methods of Enzymatic Analysis. Vol.6. 3rd ed. Deerfield Beach. Verlag Chemie, Deerfield Beach, p.178-184.

Bonel-Raposo J.P, Driemeier D., Barros S.S. \& Fernandes C.G. 2003. Evolução das lesões histológicas e ultra-estruturais no fígado de ovinos e bovinos experimentalmente intoxicados por Myoporum laetum. Pesq. Vet. Bras. 23(4):149-155.

Braga M.B., Castilhos L.M.L. \& Santos M.N. 1985. Biópsia hepática em bovinos: proposta de nova técnica. Ciência Rural, Santa Maria, 15(1):79-88.

Brum K.B., Purisco E., Lemos R.A.A. \& Riet-Correa F. 2002. Intoxicação por Vernonia rubricaulis em bovinos no Mato Grosso do Sul. Pesq. Vet. Bras. 22(3):147-148.

Canella C.F.C., Tokarnia C.H. \& Döbereiner J. 1968. Intoxicação por Sessea brasiliensis Toledo em bovinos. Pesq. Agropec. Bras. 3:333-340.

Castellani E.D. \& Aguiar I.B. 1998. Preliminary conditions for germination of Trema micrantha (L.) Blume seeds. Revta Bras. Eng. Agríc. Ambiental 2(1):80-83.

Cattani C.S., Colodel E.M., Traverso S.D., Correa A.M.R. \& Driemeier D. 2004. Intoxicação experimental por Dodonea viscosa (Sapindaceae) em bovinos. Pesq. Vet. Bras. 24(1):31-34.

Colodel E.M., Driemeier D. \& Pilati C. 2000. Intoxicação experimental pelos frutos de Xanthium cavanillesii (Asteraceae) em bovinos. Pesq. Vet. Bras. 20(1):31-38. 
Colodel E.M., Traverso S.D., Seitz A.L., Correa A.M.R., Oliveira F.N., Driemeier D. \& Gava A. 2003. Spontaneous poisoning by Dodonea viscosa (Sapindaceae) in cattle. Vet. Hum. Toxicol. 45(3):147-148.

Döbereiner J., Tokarnia C.H. \& Canella C.F.C. 1969. Intoxicação por Cestrum laevigatum Schlecht., a causa das mortandades em bovinos no Estado do Rio de Janeiro. Pesq. Agropec. Bras. 4:165-193.

Döbereiner J., Tokarnia C.H. \& Purisco E. 1976. Vernonia mollissima, planta tóxica responsável por mortandades de bovinos no sul do Mato Grosso. Pesq. Agropec. Bras. 11:49-58.

Driemeier D., Irigoyen L.F., Loretti A.P., Colodel E.M. \& Barros C.S.L 1999. Intoxicação espontânea pelos frutos de Xanthium cavanillesii (Asteraceae) em bovinos no Rio Grande do Sul. Pesq. Vet. Bras. 19(1):1-13.

Gava A. 2000. Comunicação pessoal (Laboratório de Patologia Animal, UDESC, Lages, SC).

Gava A., Stolf L., Pilati C., Neves D.S. \& Viganó L. 1991. Intoxicação por Cestrum corymbosum var. hirsutum (Solanaceae) em bovinos no Estado de Santa Catarina. Pesq. Vet. Bras. 11(3/4):71-74.

Gava A., Stolf L., Varaschin M.S., Neves D.S., Tigre A.P. \& Lessmann F. 1996. Intoxicação por Cestrum intermedium (Solanaceae) em bovinos. Pesq. Vet. Bras. 16(4):117-120.

Jubb K.V.F. \& Huxtable C.R. 1993. The nervous system, p.267-440. In: Jubb K.V.F., Kennedy P.C.\& Palmer N. (ed.) Pathology of Domestic Animals. Vol. 1. 4th ed. Academic Press, San Diego.

Maxie M.G. 1993. The urinary system, p.343-411. In: Jubb K. V. F., Kennedy P. C. \& Palmer N. (ed.) Pathology of Domestic Animals. Vol. 2. 4th ed. Academic Press, San Diego.

Mills B. 1992. Immunohistochemistry. p.247-256. In: Prophet E.B., Mills B., Arrington J.B. \& Sobin L.H. Laboratory Methods in Histotechnology. Armed Forces Institute of Pathology, Washington, DC.

Nowotny K. \& Nowotny M.P. 1993. Agrossilvicultura baseada na dinâmica e na biodiversidade da Mata Atlântica, p.11-20. In: Jesus E.L., von der
Weid J.M., Grzybowski L.M.C. \& Almeida S.G. (ed.) Alternativas. Cadernos de Agroecologia. AS-PTA, Rio de Janeiro.

Prophet E.B., Mills B., Arrington J.B. \& Sobin L.H. 1992. Laboratory Methods in Histotechnology. Armed Forces Institute of Pathology, Washington, DC. 279 p.

Radostits O.M., Gay C.C., Blood D.C. \& Hinchcliff K.W. 2000. Veterinary Medicine. 9th ed. Baillière Tindall, London. 1763 p.

Riet-Correa F., Schild A.L. \& Méndez M.C. 1986. Intoxicação por Cestrum parqui (Solanaceae) em bovinos no Rio Grande do Sul. Pesq. Vet. Bras. 6(4):111-115.

Sims F.H. \& Horn C. 1958. Some observations Powel's method for the determination of serum bilirubin. Am. J. Clin. Path. 29:412.

Szasz G. 1969. A kinetic photometric method for serum gamma-glutamyl transpeptidase Clin Chem 15: 124-136.

Tennant B. C., 1997: Hepatic function. In: Kaneko, J.J., Harvey J.W. \& Bruss M.L. (ed.), Clinical Biochemistry of Domestic Animals, 5th ed, p. 327352. Academic Press, San Diego.

Tokarnia C.H. \& Döbereiner J. 1982. Intoxicação de bovinos por Vernonia rubricaulis (Compositae) em Mato Grosso. Pesq. Vet. Bras. 2(4):143-147.

Tokarnia C.H., Döbereiner J. \& Peixoto P.V. 2000 Plantas Tóxicas do Brasil. Editora Helianthus, Rio de Janeiro. 310 p.

Traverso S.D. \& Driemeier D. 2000. Experimental Trema micrantha (Ulmaceae) poisoning in rabbits. Vet. Human Toxicol. 42(5):301-302.

Traverso S.D., Correa A.M., Pescador C.A., Colodel E.D., Cruz C.E.F. \& Driemeier D. 2002. Intoxicação experimental por Trema micrantha (Ulmaceae) em caprinos. 2002. Pesq. Vet. Bras. 22(4):141-147.

Traverso S.D., Colodel E.D., Loretti A.P., Correa A.M. \& Driemeier D. 2003. Intoxicação natural por Trema micrantha em caprinos. Ciência Rural, Santa Maria, 33(1):133-136.

Traverso S.D. \& Driemeier D. 2004. Dados não publicados (Setor de Patologia Veterinária, UFRGS, Porto Alegre, RS) 